\title{
Updates in Retinal Diseases 2018
}

\author{
An Expert Interview with Maurizio Battaglia Parodi
}

Department of Ophthalmology Ospedale San Raffaele, University Vita-Salute Milan, Italy

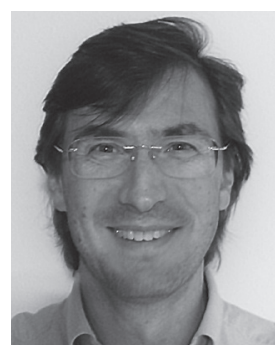

DOI: https://doi.org/10.17925/EOR.2018.12.1.19

\section{Maurizio Battaglia Parodi}

Professor Maurizio Battaglia Parodi is currently working at the Department of Ophthalmology, Scientific Institute San Raffaele in Milan and is Associate Professor at University Vita-Salute in Milan, Italy. He had previous appointments in the Departments of Ophthalmology at the University of Trieste and University of Udine, both in Italy. Professor Battaglia Parodi's major clinical and research interests include age-related macular degeneration, vascular diseases and dystrophies. He has taken part in many international clinical trials and has authored over 210 peer-reviewed scientific articles and three books. Professor Battaglia Parodi has received many awards for his scientific activity and is a reviewer for the most important international journals.

\section{Keywords}

Biomarkers, choroidal

neovascularisation, gene therapy

Disclosure: Maurizio Battaglia Parodi has nothing to disclose in relation to this article.

Acknowledgements: Medical writing support was provided by Katrina Mountfort of Touch Medical Media.

Review Process: This is an expert interview and as such has not undergone the journal's standard peer review process.

Authorship: All named authors meet the International Committee of Medical Journal Editors (ICMJE) criteria for authorship of this manuscript, take responsibility for the integrity of the work as a whole, and have given final approval to the version to be published

open Access: This article is published under the Creative Commons Attribution Noncommercial License, which permits any noncommercial use distribution, adaptation, and reproduction provided the original author(s) and source are given appropriate credit. () The Authors 2018.

Received: 20 July 2018

Published Online: 13 August 2018

Citation: European Ophthalmic Review. 2018; 12(1):19-20

Corresponding Author: Maurizio Battaglia Parodi, Department of Ophthalmology, Vita-Salute University, Ospedale San Raffaele, Via Olgettina 60, 20132, Milan, Italy. E: maubp@yahoo.it

Support: No funding was received for the publication of this article.
C horoidal neovascularisation (CNV) is a major cause of vision loss. For more than 10 years, intravitreal injections of anti-vascular endothelial growth factor (VEGF) drugs have been used in the treatment of CNV. While effective, this treatment strategy is limited by the injection burden. Most patients require multiple injections and response levels can vary. Early diagnosis and treatment of CNV can increase the success rate of treatment. There is, therefore, considerable interest in identifying biomarkers both to identify patients most likely to respond to treatment and to facilitate early detection of the condition. In an expert interview, Maurizio Battaglia Parodi of the University Vita-Salute Milan, Italy, discusses the latest advances in CNV.

\section{Q. What is currently known about biomarkers of choroidal neovascularisation?}

The intravitreal anti-vascular endothelial growth factor (VEGF) approach has proven to be effective in the treatment of CNV and diabetic macular oedema. Many treatment regimens have been proposed to make the clinical application easier. Nevertheless, there is an immense difference between randomised clinical trials and those of real-world practice. In addition to the well-known organisational issues, we know that patients can respond variably to anti-VEGF therapy. Thus, we would need to sub-classify our patients in order to customise the approach according to the specific characteristics of the CNV subtype. For that reason, some studies have focused on the identification of biomarkers related to the disease activity. Many biomarkers have been analysed, including genetic and imaging-related ones. Regarding the genetic biomarkers, the results are quite controversial, so we cannot precisely identify a genetic profile of a responder or a non-responder patient. Taking into consideration the imaging-related biomarkers, many aspects have been studied, including fundus autofluorescence, angiography, optical coherence tomography and optical coherence tomography angiography.

\section{Q. How do you see this changing/developing in the future?}

Unfortunately, the results achieved to date regarding the detection of useful biomarkers are not conclusive. I believe that in the future we will learn much more about the genetic profile and we will also be able to identify specific pharmacogenetic profiles in order to recognise non-responders a priori. As the imaging-related biomarkers, hyper-reflective foci and subretinal hyper-reflective material as identified on structural optical coherence tomography will help us better characterise CNV activity without the need of dye angiography. Promising results may also be obtained by the clinical application of optical coherence tomography angiography focusing on the vascular patterns of the CNV and its fractal dimension. 


\section{Q. Why is there a need to update intravitreal injections of anti-vascular endothelial growth factor therapy?}

In spite of the good clinical outcomes reported by randomised clinical trials on the administration of anti-VEGF drugs, we are perfectly aware of the problems related to real-world application. A number of organisational, regulatory and practical problems hamper the correct application of a powerful therapy such as intravitreal injection of anti-VEGF molecules. We need to precisely understand how an anti-VEGF approach is practically applied in our centres in an attempt to correct the critical points and to implement the final effect of the therapy. Overall, it seems from the scant data currently available in literature, that the number of injections during the first year of therapy varies from three to seven, allowing a limited functional improvement. I believe that, starting from the tangible analysis of each centre's data, we may improve the practical management of our patients, trying to simplify the administrative and organisational problems.

\section{Q. What topics are you looking forward to hearing about in the future?}

I am particularly interested in the potential application of the advancements in ophthalmic genetics. The genetic profiling of each patient could theoretically guide our therapeutic choice, reducing burden and avoiding, or at least limiting, the non-responder effect. In addition, the implementation in optical coherence tomography angiography techniques may simplify the post-processing analyses, making the interpretation of the imaging easier and quicker. I would like also to hear about the progress in robotic surgery, which can greatly help us apply complex surgical procedures, paving the way to a great diffusion of gene therapy and stem cell therapy for macular disorders. 